\title{
La competencia emocional como predictora de la felicidad en trabajadores sociales
}

\section{Emotional competence as a predictor of happiness in social workers}

\author{
Mario Millán-Franco ${ }^{1}$, Alejandro Orgambídez Ramos², \\ Laura Domínguez de la Rosa ${ }^{3}$ y Sofía Louise Martínez-Martínez ${ }^{4}$ \\ ${ }^{1}$ Doctor por la Universidad de Málaga. Graduado en Trabajo Social. Personal docente e \\ investigador del Departamento de Psicología Social, Trabajo Social, Antropología Social y \\ Estudios de Asia Oriental, área de Trabajo Social y Servicios Sociales, Facultad de Estudios \\ Sociales y del Trabajo, Universidad de Málaga, España. E-mail: mmillan@uma.es \\ ${ }^{2}$ Doctor por la Universidad de Huelva. Personal docente e investigador del Departamento de \\ Psicología Social, Trabajo Social, Antropología Social y Estudios de Asia Oriental, área de \\ Psicología Social, Facultad de Psicología y Logopedia, Universidad de Málaga. \\ E-mail: aorgambidez@uma.es \\ ${ }^{3}$ Doctora por la Universidad de Málaga. Personal docente e investigador del Departamento de \\ Psicología Social, Trabajo Social, Antropología Social y Estudios de Asia Oriental, área de \\ Trabajo Social y Servicios Sociales, Facultad de Estudios Sociales y del Trabajo, Universidad de \\ Málaga. E-mail: 1dominguez@uma.es \\ ${ }^{4}$ Graduada en Derecho. Graduada en Administración y Dirección de Empresas. Magister \\ en Sociología Aplicada. Personal investigador y doctoranda del Programa de Doctorado de \\ Economía y Empresa, Facultad de Ciencias Económicas y Empresariales, Universidad de \\ Málaga. E-mail: sofia.martinez@uma.es

\begin{abstract}
El primer autor agradece la ayuda del Plan Propio de Investigación y Transferencia de la
Universidad de Málaga para el desarrollo de una estancia de investigación posdoctoral sobre el artículo que se presenta.
\end{abstract} \\ realizada en la Universidad Nacional de Irlanda (Maynooth) en 2019, durante la cual trabajó \\ Universidad de Málaga, España
}

\section{Resumen}

Los trabajadores sociales tienen unas elevadas exigencias laborales como consecuencia, entre otros factores, de la excesiva burocratización de las organizaciones de servicios sociales. Ante esta realidad, durante su proceso formativo, los estudiantes de trabajo social deben desarrollar competencias emocionales para hacer frente a estas demandas profesionales que pueden afectar a su bienestar y rendimiento laboral. Se realizó un estudio descriptivo y correlacional de corte transversal del que participaron 187 estudiantes del primer y segundo curso $(n=105$ y $n$ $=82$, respectivamente) de Trabajo Social de la Universidad de Málaga (España). El 89.30 \% eran mujeres y la edad promedio fue de 20.38 años (DT = 3.35). Se desarrolló un modelo de regresión lineal múltiple para evaluar el papel predictor de la inteligencia emocional (atención emocional, claridad emocional y reparación de las emociones) sobre la felicidad subjetiva percibida en estudiantes de trabajo 
social. La claridad emocional y la reparación de las emociones fueron predictores significativos positivos de la felicidad subjetiva, mientras que la atención emocional fue un predictor significativo negativo. Los estudiantes y futuros trabajadores sociales tienen que entender y manejar sus estados emocionales, de tal manera que les permita tanto afrontar sus propias frustraciones como constituirse en referentes de los usuarios para imponerse a sus dificultades. Se corrobora la necesidad de fomentar la inteligencia emocional entre los estudiantes y futuros trabajadores sociales, ya que esto constituye un factor de protección que puede aumentar su bienestar y concretamente, su felicidad subjetiva. Las experiencias de afecto negativas que sufren los trabajadores sociales demandan planes de estudio en los que se fomente la inteligencia emocional. Palabras clave: servicios sociales, inteligencia emocional, trabajo social, bienestar subjetivo, felicidad subjetiva, España.

\section{Abstract}

As a result of the excessive bureaucratization of social service organizations, among other factors, social workers have many job responsibilities (López \& Chaparro, 2006; Montagud, 2016). Social workers suffer negative experiences and a high psychosocial risk which affect their well-being and work performance (i. e., emotional fatigue, job dissatisfaction, mental overload, burnout, decrease in happiness, ethical conflicts) (Bunce, Lonsdale, King, Childs, \& Bennie, 2019; Caravaca, Carrión, \& Pastor, 2018; Grootegoed \& Smith, 2018; Prada-Ospina, 2019; Sabater, De Armas, \& Cabezas, 2019; Urien, Díez, \& Osca, 2019; Vallellano \& Rubio-Valdehita, 2018).

Given this reality, it is very important that Social Work students develop, during their training process, emotional competencies to cope with such professional demands (Bedoya-Gallego, Buitrago-Duque, \& Vanegas-Arbeláez, 2019; Stanley \& Bhuvaneswari,
2016). The characteristics of the job require knowledge of the mechanisms that influence the welfare of Social Work students in order to incorporate specific training strategies into teaching plans that prepare them to face the high work demands that can affect their welfare and performance (Blakemore \& Agllias, 2019; Borrego-Alés, Vázquez-Aguado, \& Orgambídez-Ramos, 2020; Fernández-Berrocal \& Extremera, 2009). Despite the considerable shortage of research about the implications of emotional intelligence in the field of Social Work, emotional competence has proven to be essential to explain the personal well-being of students and social intervention professionals, characterized by a high burden of work and high rates of psychological distress and burnout (Bae et al., 2020; Esteban, 2014; Mikulic, Crespi, \& Radusky, 2015).

A descriptive and correlational cross-sectional study was carried out. 187 students of first $(\mathrm{n}=105)$ and second year $(\mathrm{n}=82)$ of the University of Malaga's Social Work Degree (Spain) participated. $89.30 \%$ of them was women and the average age was 20.38 years $(\mathrm{SD}=3.35)$. A multiple linear regression model evaluated the predictive role of emotional intelligence (attention, clarity and emotional repair) on the subjective happiness perceived in Social Work students. Emotional clarity and emotional repair were significant positive predictors of subjective happiness, while emotional attention was a significant negative predictor. Of the three independent variables, emotional repair was positioned as the strongest predictor of the perception of subjective happiness. This fact corroborates that emotional repair is especially important to predict the levels of subjective happiness and life satisfaction of university students (Cejudo, López-Delgado, \& Rubio, 2016; Extremera, Salguero, \& Fernández-Berrocal, 2011).

High levels of emotional repair are essential for proper psychological functioning and mental health. In this sense, people tend to develop pleasant activities that distract 
them and therefore contribute to their happiness (Guerra-Bustamante, León-del-Barco, Yuste-Tosina, López-Ramos, \& Mendo-Lázaro, 2019). Individuals who can clearly identify their emotions and believe that they can repair their negative moods minimize the impact of stressful events and generate more positive emotions (Extremera et al., 2011). In social workers, emotions can be understood both as a dynamic resource, whose research can protect professionals and users, and as patterns of organizational and professional relationships, in which emotional states are constituted as phenomena that can be managed and controlled during professional practice (O’Connor, 2020).

The need to foster emotional intelligence among students and future social workers is confirmed. It constitutes a protective factor that can increase their well-being and, specifically, their subjective happiness. The negative experiences of affection that social workers suffer demand curricula that promote emotional intelligence. The students and future social workers have to understand and manage their emotional states. It allows them both to face their own frustrations and to become referents for the users when they overcome their difficulties.

Keywords: social services, emotional intelligence; social work, subjective well-being, subjective happiness, Spain.

\section{Introducción}

Los trabajadores sociales tienen unas elevadas demandas laborales como consecuencia de la excesiva burocratización de las organizaciones de servicios sociales (López y Chaparro, 2006; Montagud, 2016; Soto-Rosales y González-Losada, 2018). En este sentido, los trabajadores sociales sufren experiencias negativas y un elevado riesgo psicosocial que afectan a su bienestar y a su propio rendimiento laboral $(i$. e., cansancio emocional, insatisfacción laboral, sobrecarga mental, burnout, disminución de felicidad, conflictos éticos) (Bunce et al., 2019; Caravaca et al., 2018; Grootegoed y Smith, 2018; Prada-Ospina, 2019; Sabater et al., 2019; Urien et al., 2019; Vallellano y Rubio-Valdehita, 2018). Ante esta realidad es de vital importancia que durante su proceso formativo, los estudiantes de trabajo social desarrollen competencias emocionales para hacer frente a dichas exigencias profesionales que pueden afectar sus niveles de bienestar $y$ rendimiento laboral (Bedoya-Gallego et al., 2019; Stanley y Bhuvaneswari, 2016). De la misma forma, las características del puesto de trabajo demandan conocer los mecanismos que influyen en el bienestar de los estudiantes de trabajo social para poder incorporar estrategias formativas concretas en los planes docentes que los preparen para afrontar las elevadas exigencias laborales y les permita mantener unos niveles adecuados de calidad de vida y optimizar su actividad profesional (Blakemore y Agllias, 2019; Borrego-Alés et al., 2020; Fernández-Berrocal y Extremera, 2009).

\section{La importancia de la competencia emocional en la práctica del trabajo social}

A pesar de la considerable escasez de investigaciones en estudiantes y profesionales sobre las implicaciones de la inteligencia emocional en el ámbito del trabajo social, la competencia emocional ha demostrado ser fundamental para explicar el bienestar personal de los profesionales de la intervención social, caracterizados por una alta carga de trabajo y elevados índices de angustia psicológica y burnout (i. e., satisfacción por compasión, engagement, satisfacción con la vida, felicidad subjetiva) (Bae et al., 2020; Extremera, Durán y Rey, 2005; Mayer, Salovey y Caruso, 2008; Mikulic et al., 2015; Morilla-Luchena, Borrego-Alés, Orgambídez-Ramos y Vázquez-Aguado, 2019). Los trabajadores sociales que poseen una adecuada inteligencia emocional se encuentran mejor preparados 
para mantener una buena calidad de vida laboral y una apropiada felicidad subjetiva, lo cual contribuye a su vez al desarrollo de la relación de servicio con el usuario (Dore, 2019; Ingram, 2013). Así, recientemente en el contexto del trabajo social, se ha manifestado la importancia de la emoción tanto como práctica relacional como un recurso que informa de los procesos de creación de sentido en las relaciones entre profesionales y en la propia intervención con los usuarios (O’Connor, 2020). Debido a la relevancia de las relaciones en la práctica profesional de los trabajadores sociales, el concepto de inteligencia emocional cobra especial interés para la formación y actuación de este colectivo en distintos ámbitos (Stanley, Buvaneswari y Meenakshi, 2020). En este sentido, Morrison (2007) considera la importancia de la competencia emocional en cinco aspectos centrales de la intervención de los trabajadores sociales: (1) engagement o ilusión hacia el trabajo; (2) valoración y observación; (3) toma de decisiones; (4) colaboración y cooperación; y (5) manejo del estrés, desarrollo de resiliencia y estrategias de afrontamiento.

Dicho esto, lo interpersonal, el corazón y lo intelectual, la cabeza, se han considerado relevantes para una óptima competencia laboral y las materias primas de las que surge un buen trabajador social (Sheppard y Charles, 2015). Si bien todavía se desconocen las potenciales relaciones y las tensiones entre ambos dominios, se ha señalado que la inteligencia emocional puede ser el nexo de unión entre ambas competencias (Sheppard, Charles, Rees, Wheeler y Williams, 2018).

La habilidad para gestionar las reacciones emocionales en entornos de atención complejos, i. e., procesos migratorios o situaciones de necesidad, es fundamental para los trabajadores sociales, por lo que la inteligencia emocional puede ayudar a los estudiantes y futuros profesionales a manejar sus reacciones emocionales de manera más efectiva (Grant, Kinman y Alexander, 2014; Millán-Franco, Gómez-Jacinto, Hombrados-
Mendieta, García-Martín y García-Cid, 2019; O'Connor, 2020; Sewell, 2020). De la misma forma, en la visita domiciliaria, entendida como una técnica inherente al trabajo social, la competencia emocional juega un papel fundamental, por lo que las respuestas emocionales de los profesionales durante este proceso tienen grandes implicaciones en el éxito de la intervención social (Cook, 2020). En definitiva, los estudiantes de trabajo social, como futuros profesionales, tienen que entender y manejar sus estados emocionales, de tal manera que puedan tanto afrontar sus propias frustraciones como constituirse en referentes de los usuarios a la hora de imponerse a sus dificultades (Golightley y Kirwan, 2019).

\section{Conceptualización y operacionalización de la inteligencia emocional y la felicidad subjetiva}

La noción de inteligencia emocional ha sido desarrollada considerablemente en los últimos tiempos, y se han propuesto diversas escalas para medirla (Stanley et al., 2020). Esta puede entenderse como la competencia para controlar las propias emociones, la habilidad para influir en las emociones de los otros y el uso de las emociones en contextos de resolución de problemas (Bae et al., 2020). Dicho esto, Salovey y Mayer (1990) presentan un modelo teórico empíricamente demostrado sobre la concepción y manifestación de la inteligencia emocional en un conjunto de competencias o habilidades: (1) la percepción emocional o destreza en la identificación de las emociones tanto propias como ajenas; (2) la facilitación emocional o la destreza para utilizar las emociones para apoyar el razonamiento; (3) la comprensión emocional o destreza para resolver problemas y reconocer emociones similares; y (4) la dirección emocional o destreza para entender los alcances de los hechos sociales en las emociones y la regulación de las emociones propias y ajenas.

Posteriormente, Salovey, Mayer, Goldman, Turvey y Palfai (1995), al proponer la noción 
de meta-mood experience, presentan la Trait Meta-Mood Scale (TMMS). Esta recoge tres dimensiones claves en la conceptualización y evaluación de la inteligencia emocional: (1) la atención de las emociones o el nivel en el que un sujeto es consciente, vigila y delibera sobre sus estados de ánimo y emociones; (2) la claridad emocional o el nivel en el que un sujeto conoce y comprende sus estados de ánimo; y (3) la reparación de las emociones o el nivel en que una persona regula y modera sus emociones. Desde esta perspectiva, el rasgo de la inteligencia emocional se refiere a una disposición conductual que incluye la capacidad de participar en el procesamiento complejo de información sobre las emociones propias y ajenas, y la capacidad de utilizar esta información para guiar el pensamiento y el comportamiento, cumpliendo funciones adaptativas fundamentales para el bienestar de los individuos (Mayer et al., 2008; Ye, Yeung, Liu y Rochelle, 2019).

En las últimas décadas se ha apreciado un aumento importante de la investigación sobre la felicidad y sus relaciones con el bienestar, la calidad de vida laboral y los estados emocionales positivos de los diferentes colectivos profesionales que desarrollan intervención social (Sánchez-Álvarez, Extremera y Fernández-Berrocal, 2016). La felicidad se puede entender como un estado emocional, fruto de la interacción de los diferentes condicionantes que operan sobre los sujetos, que genera respuestas que ejercen resultados positivos en diversos ámbitos de la vida $\mathrm{y}$, en última instancia, acaba beneficiando al conjunto de la sociedad (i. e., autorrealización con el trabajo) (Pulido y Herrera, 2018). En este sentido, la felicidad subjetiva se concibe más duradera que los estados de ánimo momentáneos o diarios, aunque es modificable $\mathrm{y}$, por tanto, es factible su desarrollo a medio o largo plazo (Extremera y Fernández-Berrocal, 2014). Desde esta perspectiva, en palabras de Diener, Suh, Lucas y Smith (1999) la felicidad se materializa en la conjunción de tres dimensiones interrelacionadas: (1) el afecto negativo escasamente habitual; (2) los casos habituales de afecto positivo; y (3) una elevada satisfacción vital.

\section{El efecto de la inteligencia emocional en las variables psicosociales que influyen en el bienestar de los trabajadores sociales}

Tal y como se ha señalado, debido al énfasis en la práctica basada en las relaciones, la competencia emocional es de relevancia para los estudiantes y futuros trabajadores sociales. Niveles elevados de inteligencia emocional están asociados con una amplia gama de resultados intrapersonales positivos y se ha señalado que esta es un apropiado predictor del afrontamiento adaptativo ante las adversidades de la vida (Gómez-Romero, Limonero, Toro, Montes-Hidalgo y Tomás-Sábado, 2018). En estudiantes de trabajo social la inteligencia emocional, concretamente la evaluación y la expresión de las emociones, es un predictor positivo significativo de la resiliencia (Bunce et al., 2019; Stanley y Bhuvaneshwari, 2018); la claridad emocional es predictora de la capacidad de solucionar problemas sociales $\mathrm{y}$, en parte, la orientación negativa hacia los problemas puede explicarse por altos niveles de atención emocional (Augusto, Aguilar-Luzón y Salguero, 2008).

Las habilidades efectivas para el manejo de las emociones pueden ayudar a presentes y futuros trabajadores sociales a resistir la naturaleza estresante de su profesión (Grant y Kinman, 2012; Samper-García, Mesurado, Richaud y Llorca, 2016), y se señala que los trabajadores sociales logran mejorar sus niveles de resiliencia individual a través de una elevada claridad emocional (Crowder y Sears, 2017). En profesionales del trabajo social en España la claridad emocional se ha manifestado como predictora significativa de la dimensión realización personal del burnout $\mathrm{y}$ de las tres dimensiones del engagement (vigor, dedicación y absorción), mientras que la reparación emocional ha sido predictora 
de las dimensiones dedicación y absorción del engagement y agotamiento emocional del burnout (Esteban, 2014). De esta forma, en términos generales, se observa cómo en trabajadores sociales la claridad y la reparación de las emociones poseen una relación directa con los indicadores de calidad de vida laboral $(i$. e., ilusión por el trabajo) (Esteban-Ramiro y Fernández-Montaño, 2017).

Actualmente las explicaciones disposicionales de la felicidad enfatizan la importancia de la inteligencia emocional, y se señala que esta puede predecir la felicidad más allá de la influencia de los cinco grandes rasgos de personalidad (apertura a la experiencia, responsabilidad, extroversión, amabilidad e inestabilidad emocional o neuroticismo) (Callea, De Rosa, Ferri, Lipari y Costanzi, 2019; Chamorro-Premuzic, Bennett y Furnham, 2007). Así, se ha identificado la inteligencia emocional como predictora del bienestar y de la felicidad subjetiva de estudiantes universitarios (Extremera \& Rey, 2016; Ruiz-Aranda, Extremera, \& Pineda-Galán, 2014; Ye et al., 2019). Recientes metaanálisis han ratificado la relación significativa positiva entre la inteligencia emocional y el bienestar subjetivo de este colectivo (Sánchez-Álvarez et al., 2016).

Resultados en adolescentes residentes en España han mostrado que, a medida que aumenta la claridad y la reparación de las emociones, los sujetos se perciben más felices, mientras que la atención emocional no evidenció asociación (Guerra-Bustamante et al., 2019). Dificultades en la regulación de las emociones, una desmedida atención emocional, una insuficiente claridad emocional e incapacidad para solicitar ayuda cuando se necesita predicen comportamientos suicidas en adolescentes argentinos (Galarza, Castañeiras y Fernández, 2018). En investigaciones con estudiantes universitarios del sur de España, la claridad emocional predijo el bienestar psicológico, el optimismo y el pesimismo; la atención emocional, el optimismo y el pesimismo, y la regulación emocional, los niveles de optimismo y el pesimismo disposicional
(Augusto-Landa, Pulido-Martos y LopezZafra, 2011). En este sentido, se ha señalado que la reparación emocional puede contribuir a predecir la felicidad subjetiva de estos universitarios (Extremera et al., 2011). De la misma forma, en estudiantes universitarios en territorio español, los altos niveles de reparación emocional predijeron la satisfacción con la vida, mientras que elevadas puntuaciones en atención emocional se asociaron negativamente con la satisfacción vital (Cejudo et al., 2016). En profesionales de la intervención social en el sur de España, los sujetos con índices elevados de claridad y reparación emocional y una atención emocional moderada presentaron mayores niveles de felicidad subjetiva (Extremera et al., 2005).

\section{Interés y objetivo del estudio}

Dicho esto, hasta la fecha existe escasa investigación sistemática que haya explorado las implicaciones de las emociones y la inteligencia emocional en el bienestar de estudiantes y profesionales del trabajo social (Grant et al., 2014; Ibrahim, Jarimal, Nawi y Ahmad, 2018; O'Connor, 2020). Concretamente, en estudiantes y trabajadores sociales las investigaciones sobre las relaciones entre las dimensiones de la inteligencia emocional y la felicidad subjetiva siguen siendo residuales, especialmente en territorio español (Stanley et al., 2020). El objetivo principal de esta investigación es evaluar el rol predictor de las dimensiones de la inteligencia emocional (atención emocional, claridad emocional y reparación de las emociones) sobre la felicidad subjetiva percibida en una muestra de estudiantes de Trabajo Social de una universidad pública del sur de España. En este sentido, se plantean las siguientes hipótesis:

H1a: La atención emocional es un predictor significativo de la felicidad subjetiva

H1b: La claridad emocional es un predictor significativo de la felicidad subjetiva 
H1c: La reparación de las emociones es un predictor significativo de la felicidad subjetiva

\section{Metodología}

\section{Diseño de investigación, muestreo y recolección de datos}

Se realizó un estudio de carácter exploratorio, descriptivo y correlacional de corte transversal. Se llevó a cabo un muestreo por conveniencia utilizándose los siguientes criterios de selección: (1) Estar matriculado en Trabajo Social en la Universidad de Málaga (España); (2) ser estudiante de primer o segundo curso; (3) no haber recibido ninguna formación específica en competencias emocionales. Participaron 187 estudiantes de primer año $(n=105)$ y segundo curso $(\mathrm{n}=82)$ de Trabajo Social de la Universidad de Málaga (España). En cuanto a las características sociodemográficas de la muestra, el $89.30 \%$ eran mujeres y la edad media de los participantes se situó en 20.38 años $(\mathrm{DT}=3.35)$. En función de la naturaleza de las hipótesis de investigación, se desarrolló un modelo de regresión lineal múltiple para evaluar el papel predictor de la atención emocional, la claridad emocional y la reparación emocional sobre la percepción de felicidad subjetiva.

La información se recopiló entre marzo y abril de 2019. Los cuestionarios se aplicaron al comienzo de la clase, cediendo a los estudiantes todo el tiempo necesario para completarlos. Se les informó sobre los objetivos de la investigación y la confidencialidad de sus datos, y se les solicitó su consentimiento para colaborar. A cada uno de los participantes se le proporcionó un documento en el que se incluía la descripción de la investigación y una serie de normas generales, la compilación de los instrumentos (Trait Meta-Mood Scale, TMMS y Subjective Happiness Scale, SHS) e información sociodemográfica relevante (sexo, edad, curso). Durante el desarrollo de la investigación se respetaron todos los principios éticos de la National Association of Social Workers (NASW, 2017) y de la American Psychological Association (APA, 2019).

Se utilizó el paquete estadístico STATA v.13 para desarrollar el análisis de datos. Los estadísticos descriptivos obtenidos fueron la media, la desviación típica, la curtosis y la asimetría. Se comprobó que se cumplían los supuestos de normalidad, multicolinealidad y homocedasticidad de manera previa a la ejecución del análisis. Las correlaciones entre las variables se obtuvieron utilizando la correlación de Pearson (nivel de significatividad de .05 , bilateral) y los coeficientes de fiabilidad de las escalas se calcularon a través del alpha de Cronbach. La regresión lineal múltiple se utilizó para evaluar la capacidad de la atención emocional, la claridad emocional y la reparación de las emociones para predecir los niveles de felicidad subjetiva percibida.

\section{Instrumentos}

La inteligencia emocional fue medida a través de la Trait Meta-MoodScale (TMMS) de Salovey et al. (1995), en su versión en castellano de Fernández-Berrocal, Extremera y Ramos (2004). Los 24 ítems se distribuyen en tres dimensiones de ocho ítems cada una: atención emocional, claridad emocional y reparación de las emociones. Las respuestas de todos los ítems se realizan a través de una escala tipo Likert de 1 (nada de acuerdo) a 5 (totalmente de acuerdo). La fiabilidad de los instrumentos (alpha de Cronbach) se situó en todos los casos por encima de lo recomendable: atención emocional (.86), claridad emocional (.90) y reparación de las emociones (.84).

La felicidad subjetiva fue evaluada por medio de la Subjective Happiness Scale (SHS) de Lyubomirsky y Lepper (1999), versión en castellano, de Extremera y Fernández-Berrocal (2014). El instrumento consta de cuatro ítems y todas las respuestas se realizan a través de una escala tipo Likert que varía entre 1 y 7 . En general, me considero (1. Una 
persona no muy feliz - 7. Una persona muy feliz), 2. Comparado con la mayoría de la gente que me rodea, me considero (1. Menos feliz - 7. Más feliz), 3. Algunas personas suelen ser muy felices. Disfrutan la vida a pesar de lo que ocurra, afrontando la mayoría de las cosas. ¿En qué medida te consideras una persona así? (1. Nada en absoluto - 7. En gran medida), 4. Algunas personas suelen ser muy poco felices. Aunque no están deprimidas, no parecen tan felices como ellas quisieran. ¿En qué medida te consideras una persona así? (1. Nada en absoluto - 7. En gran medida). La fiabilidad de la felicidad subjetiva ( $\alpha$ de Cronbach) fue adecuada (.81).

\section{Resultados}

La Tabla 1 presenta un resumen de los estadísticos descriptivos y de las correlaciones desarrolladas. La media de felicidad subjetiva se situó en $5.28(D T=1.04)$, mientras que para las dimensiones de inteligencia emocional de mayor a menor fueron: atención emocional $3.66(D T=.74)$, reparación de las emociones
$3.40(D T=.76)$ y claridad emocional 3.32 $(D T=.83)$. En este sentido, se observa cómo la muestra evidenció altas puntuaciones en el conjunto de variables estudiadas, demostrándose altos niveles de felicidad subjetiva, atención emocional, claridad emocional y reparación de las emociones.

De la misma forma, se observó una relación significativa y positiva entre dos de las dimensiones de la inteligencia emocional (claridad, reparación) y la felicidad subjetiva. En base a Cohen (1988), los respectivos análisis, a través del coeficiente de correlación de Pearson, evidenciaron una correlación significativa, mediana y positiva, entre la felicidad subjetiva y la claridad emocional $(r=.33, p<.01)$ y aún más intensa entre la felicidad subjetiva y la reparación de emociones $(r=.50, p<$ $.01)$. Sin embargo, para el caso de atención emocional no se observó dicha relación $(r=$ $-.13, n . s)$. Se concluye que las altas puntuaciones en claridad y reparación emocional estuvieron asociadas con niveles elevados de felicidad subjetiva.

Tabla 1

Estadísticos descriptivos y correlaciones de todas las variables del estudio.

\begin{tabular}{c|c|c|c|c} 
& $\mathbf{1}$ & $\mathbf{2}$ & $\mathbf{3}$ & $\mathbf{4}$ \\
\hline 1. Atención emocional & $(.86)$ & & & \\
2. Claridad emocional & .08 & $(.90)$ & & \\
3. Reparación de las emociones & -.01 & $.39 * *$ & $(.84)$ & \\
4. Felicidad subjetiva & -.13 & $.33^{* *}$ & $.50^{* *}$ & $(.81)$ \\
Media & 3.66 & 3.32 & 3.40 & 5.28 \\
Desviación Típica & .74 & .83 & .76 & 1.04 \\
Curtosis & -.05 & -.70 & -.49 & .86 \\
Asimetría & -.45 & .04 & -.13 & -.88
\end{tabular}

Nota: En la diagonal, los coeficientes de fiabilidad entre paréntesis.

$* * p<.01$

Se llevó a cabo un modelo de regresión lineal múltiple donde las variables (atención emocional, claridad emocional y reparación de las emociones) se consideraron como variables predictoras y la felicidad subjetiva como variable resultado. Los supuestos de norma- 
lidad, multicolinealidad y homocedasticidad se comprobaron antes de la realización del análisis. En la Tabla 2 se presenta el modelo de regresión lineal múltiple de las dimensiones de inteligencia emocional sobre la felicidad subjetiva. El modelo de regresión resultó ser estadísticamente significativo: $F$ (3.182) $=$ $24.63, p<.01$. La varianza total explicada por el modelo fue del $27.70 \%$. Todos los coeficientes $V I F$ de las variables predictoras fueron inferiores a 10. Las tres dimensiones de inteli- gencia emocional se mostraron como predictoras significativas de la felicidad subjetiva, con los siguientes valores: $\beta=-.14(p<.05)$ para atención emocional, $\beta=.17(p<.05)$ para claridad emocional, y $\beta=.43(p<.01)$ para reparación de emociones. De esta forma, se confirmaron todas las hipótesis del estudio (H1a, H1b, H1c). De las tres variables independientes, la reparación de las emociones se mostró como el predictor más potente de la felicidad subjetiva percibida.

Tabla 2

Modelo de regresión lineal múltiple sobre felicidad subjetiva.

\begin{tabular}{c|c|c|c|c|c|c} 
& Coef. & SE & $\boldsymbol{t}$ & $\boldsymbol{p}$ & $\boldsymbol{\beta}$ & $\boldsymbol{V I F}$ \\
\hline $\begin{array}{c}\text { Atención } \\
\text { emocional }\end{array}$ & -.19 & .09 & -2.21 & .03 & -.14 & 1.19 \\
$\begin{array}{c}\text { Claridad } \\
\text { emocional }\end{array}$ & .21 & .09 & 2.49 & .01 & .17 & 1.19 \\
$\begin{array}{c}\text { Reparación de } \\
\text { las emociones }\end{array}$ & .59 & .09 & 6.32 & .00 & .43 & 1.01 \\
CTE & 3.27 & .46 & 7.15 & .00 & - & -
\end{tabular}

$F(3,182)=24.63, p<.01$, con $R^{2}$ ajustado de $.2770(27.70 \%)$

\section{Discusión}

Las elevadas exigencias profesionales a las que se enfrentan los trabajadores sociales hacen de este un colectivo muy vulnerable a los riesgos psicosociales (i.e., disminución del bienestar, reducción del rendimiento laboral) (Caravaca et al., 2018; López y Chaparro, 2006; Montagud, 2016; Prada-Ospina, 2019). Es necesario que durante su formación, los estudiantes y futuros profesionales del trabajo social desarrollen competencias emocionales para afrontar dichas demandas laborales, por lo que previamente es imprescindible conocer los mecanismos específicos que influyen en su bienestar. El objetivo fundamental de esta investigación fue comprobar la función predictora de las dimensiones de la inteligencia emocional (atención emocional, claridad emocional y reparación emocional) sobre la felicidad subjetiva percibida de estudiantes de Trabajo Social de la Universidad de Málaga.

Los resultados del modelo de regresión mostraron que la claridad emocional y la reparación de las emociones son predictores significativos positivos de la felicidad subjetiva, mientras que la atención emocional es un predictor significativo negativo. En este sentido, las tres hipótesis de la investigación (H1a, H1b, H1c) pudieron ser confirmadas. Los estudiantes con elevados niveles de claridad y reparación de las emociones y moderados de atención emocional presentaron mayores índices de felicidad subjetiva percibida. Estos hallazgos son similares a otros estudios con profesionales de la intervención social en el sur de España, en donde se manifestó la importancia de la adecuación de los niveles de las tres dimensiones de la inteli- 
gencia emocional para la positiva percepción de felicidad subjetiva de estos colectivos (Extremera et al., 2005). De la misma forma, en adolescentes argentinos se ha señalado que problemas en la regulación de las emociones, una excesiva atención emocional y una escasa claridad emocional predicen comportamientos suicidas (Galarza et al., 2018). Cada una de las emociones tiene una función positiva y las situaciones que causan molestias son inevitables, por lo que la percepción de felicidad subjetiva no depende de la ausencia de situaciones incómodas, sino del equilibro entre lo agradable y lo desagradable (Guerra-Bustamante et al., 2019).

En la presente investigación las tres dimensiones de inteligencia emocional se mostraron como predictoras significativas de la felicidad subjetiva, apoyando estudios previos que han señalado que la inteligencia emocional predice la felicidad, incluso controlando el efecto de los cinco grandes rasgos de la personalidad (Callea et al., 2019; Chamorro-Premuzic et al., 2007). Los hallazgos de este trabajo corroboran las investigaciones con estudiantes universitarios en donde la inteligencia emocional se mostró como predictora del bienestar y la felicidad subjetiva (Extremera y Rey, 2016; Ruiz-Aranda et al., 2014; Sánchez-Álvarez et al., 2016; Ye et al., 2019). Desde este planteamiento, el rasgo de inteligencia emocional cumple funciones adaptativas fundamentales para la felicidad de las personas, incluyendo la capacidad de participar en el procesamiento complejo de información sobre las emociones y la capacidad de utilizar esta información para guiar el pensamiento y el comportamiento (Ye et al., 2019).

De esta forma, una adecuada inteligencia emocional está asociada intensamente en estudiantes y trabajadores sociales con un amplio espectro de resultados intrapersonales positivos ( $i$. e., resiliencia, engagement, felicidad subjetiva), siendo predictora del afrontamiento adaptativo ante las vicisitudes de la vida (Bunce et al., 2019; Esteban, 2014; Gómez-Romero et al., 2018; Stanley y
Bhuvaneshwari, 2018). Los sujetos emocionalmente inteligentes son capaces de entender las emociones generadas en su contexto, algo fundamental para los presentes y futuros trabajadores sociales, comprendiendo sus causas y consecuencias y, en base a ello, implementar estrategias para reparar y controlar los estados emocionales que pueden generar efectos psicosociales negativos (Esteban, 2014; Mikulic et al., 2015).

Los hallazgos de este estudio señalan que la claridad emocional y especialmente la reparación de las emociones son predictoras positivas significativas de la felicidad subjetiva de los estudiantes de Trabajo Social de la Universidad de Málaga. Estos resultados sugieren que, a medida que aumenta la capacidad de comprensión y regulación de las emociones, también aumenta la felicidad subjetiva percibida. En este sentido, los resultados obtenidos son coherentes con otras investigaciones desarrolladas en territorio español, donde se comprobó que los adolescentes se perciben más felices cuando evidencian niveles elevados de claridad y reparación emocional (Guerra-Bustamante et al., 2019) y que en las estudiantes universitarias, la claridad y la regulación emocional se asocian positivamente con los componentes del bienestar psicológico (Augusto-Landa et al., 2011).

La influencia positiva de la claridad y la reparación emocional sobre la calidad de vida laboral de los propios trabajadores sociales ha sido ratificada en relación a la ilusión por el trabajo (Esteban-Ramiro y Fernández-Montaño, 2017). De la misma forma, el rol de la claridad emocional para predecir resultados positivos en estudiantes de trabajo social y trabajadores sociales se ha comprobado, entre otros, en la capacidad para resolver problemas sociales, en los niveles de resiliencia individuales y en las dimensiones del engagement (Augusto et al., 2008; Crowder y Sears, 2017; Esteban, 2014). Así, los estudiantes y trabajadores sociales con mayor capacidad para comprender sus emociones en situaciones estresantes dedicarán menos tiempo aten- 
diendo a sus reacciones emocionales, manteniendo sus pensamientos en otras áreas que bien le generen bienestar o bien le permitan desarrollar estrategias de afrontamiento y reparación emocional más adaptativas (Augusto-Landa et al., 2011; Samper-García et al., 2016).

El hecho de que de las tres variables independientes, la reparación emocional se situara como la predictora más fuerte de la percepción de felicidad subjetiva, corrobora que en estudiantes universitarios la reparación de las emociones es especialmente importante para predecir sus niveles de felicidad subjetiva y de satisfacción vital (Cejudo et al., 2016; Extremera et al., 2011). Unos elevados niveles de reparación emocional son fundamentales para el funcionamiento psicológico apropiado y la salud mental de las personas, las cuales tienden a desarrollar actividades placenteras que las distraen y por tanto, contribuyen a su felicidad (Guerra-Bustamante et al., 2019). En esta misma línea, en trabajadores sociales se ha ratificado la trascendencia de la reparación de las emociones para predecir positivamente elementos tan importantes para el bienestar del colectivo como el engagement o el propio burnout (Esteban, 2014). Los individuos que pueden identificar claramente sus emociones y creen que pueden reparar sus estados de ánimo negativos minimizan el impacto de los eventos estresantes y generan emociones más positivas (Extremera et al., 2011).

Los resultados muestran que la atención de las emociones es un predictor significativo negativo de la felicidad subjetiva percibida en los estudiantes de trabajo social. Estos hallazgos están en sintonía con estudios previos de universitarios en España, en donde una excesiva atención emocional se asoció negativamente con la satisfacción con la vida (Cejudo et al., 2016). Concretamente, en estudiantes de trabajo social, un aumento de la atención emocional explicó parte de la varianza de la orientación negativa hacia los problemas, lo que plantea que quienes evidencian elevados niveles de atención emocional tienden a perpetuar sus estados de ánimo negativos (Augusto et al., 2008). En este sentido, aunque la atención emocional es necesaria para la adaptación del sujeto al ambiente, una excesiva atención se asocia con elementos incompatibles con la felicidad como la ansiedad, la rumiación y la catastrofización (Guerra-Bustamante et al., 2019). Se corrobora la necesidad de fomentar el cultivo de la inteligencia emocional entre los estudiantes y futuros trabajadores sociales, ya no solo como factor de protección que puede aumentar su bienestar, sino también como una habilidad práctica relevante que puede mejorar la eficacia de su intervención profesional ante las elevadas demandas profesionales (Bae et al., 2020).

En la formación de los estudiantes de trabajo social, generalmente no se ha dado la atención que merece al desarrollo de las competencias relacionadas con el manejo y la regulación emocional, sobre todo teniendo en cuenta que la práctica del trabajo social es emocionalmente exigente y que la carencia de esta competencia tiene un efecto negativo en la calidad de la actividad profesional (Stanley y Bhuvaneswari, 2016; Stanley et al., 2020). Así, es necesario aumentar los contenidos enfocados al desarrollo de la competencia emocional en los planes formativos de trabajo social (Bae et al., 2020; Bedoya-Gallego et al., 2019).

Entre las limitaciones del estudio se señala, en primer lugar, el desarrollo de un muestreo por conveniencia, por lo que no permite la generalización de los resultados. En segundo término, el diseño transversal impide atribuir relaciones de causalidad entre las variables analizadas. De esta forma, el enfoque transversal se constituye como una foto que no puede describir totalmente la realidad. En tercera instancia, el uso de cuestionarios conlleva la existencia de ciertos sesgos en las respuestas (i. e., deseabilidad social, fatiga) que pueden llegar a sobredimensionar los resultados.

Dado que los resultados de este estudio son una aproximación exploratoria al objeto de 
investigación, futuras líneas de investigación deberían ser desarrolladas con mayor fundamentación empírica y orientadas a subsanar algunas de las limitaciones anteriormente expuestas. Por ejemplo, diseños que incluyan a los mismos sujetos durante su formación $\mathrm{y}$, posteriormente, tras su inserción laboral serían ciertamente interesantes. Además, en futuros estudios sería relevante detectar, a través de modelos de regresión curvilínea, el nivel de atención emocional óptimo para los diversos indicadores de calidad de vida laboral, rendimiento laboral y bienestar ( $i$. $e$, felicidad subjetiva) de presentes y futuros trabajadores sociales. En este sentido, si bien unos elevados niveles de atención emocional han demostrado ser predictores significativos de una disminución de la felicidad subjetiva, niveles demasiado reducidos de atención emocional deberían ser contraproducentes, ya que el sujeto no sería capaz de ser consciente de su propio estado emocional y actuar en base a él para repararlo. De la misma forma, en el futuro sería interesante analizar el comportamiento de estas variables y otras similares, ya no solo en estudiantes, sino propiamente en profesionales del trabajo social.

En base al éxito que proporciona la competencia emocional en el ámbito individual, grupal y organizativo del trabajo social, son evidentes sus implicaciones en el contexto educativo (Dore, 2019). Así, especialmente en el nivel de educación superior existe un vacío en la preparación de los estudiantes para desarrollar las habilidades propias de la inteligencia emocional, claves para su éxito personal y para su adecuación a las demandas laborales. Los estudiantes y futuros trabajadores sociales deben conocer y manejar las emociones, posibilitándoles tanto el afrontamiento de sus propias frustraciones, como servir de estímulo a los usuarios para superar sus dificultades. En el campo del trabajo social las emociones son un aspecto clave en el desarrollo del compromiso, la observación, la toma de decisiones, la planificación y la intervención con el usuario, por lo que durante el proceso formativo, la puesta en valor de las relaciones sociales y de las emociones debe ser una preocupación central para la disciplina (Morrison, 2007). Las frecuentes experiencias de afecto negativas, tales como la ansiedad o el dolor, a las que se ven expuestos los profesionales del trabajo social durante su actividad laboral, demandan que los planes de estudio integren programas preventivos en donde se fomente el desarrollo de la inteligencia emocional (Fernández-Berrocal y Extremera, 2009).

Entre las propuestas de intervención para mejorar el nivel de competencia emocional de los estudiantes y futuros trabajadores sociales, se señalan el desarrollo de talleres para potenciar las habilidades emocionales en los primeros años de formación y la elaboración de portafolios en donde los estudiantes realicen registros reflexivos en torno a sus estados emocionales (Grant et al., 2014). Además, la simulación y el juego de roles pueden mejorar las capacidades de regulación emocional de los estudiantes de trabajo social y prepararlos para las elevadas exigencias laborales de su práctica profesional (Sewell, 2020). Aunque el enfoque de este estudio se centra en el análisis y posterior desarrollo de competencias personales en los estudiantes y futuros trabajadores sociales -inteligencia emocional-, no se deben obviar las causas estructurales del estrés en el ámbito de los servicios sociales y por tanto, las consecuencias de las condiciones de trabajo patógenas, incluso en trabajadores sociales emocionalmente inteligentes (Grant y Kinman, 2012).

En conclusión, los resultados indican que una parte importante de la felicidad subjetiva percibida de los estudiantes y futuros trabajadores sociales puede ser predicha a partir de los niveles de atención, claridad y reparación emocional. Así, se manifiesta la pertinencia de fomentar el desarrollo de su inteligencia emocional para aumentar su felicidad subjetiva y, en última instancia, para que probablemente se puedan adaptar mejor a las vicisitudes de la vida profesional y personal (Callea 
et al., 2019). En los trabajadores sociales las emociones pueden entenderse tanto como un recurso dinámico, cuya investigación puede proteger a profesionales y usuarios, como patrones de relaciones organizacionales $\mathrm{y}$ profesionales en los que los estados emocionales se constituyen como fenómenos que pueden ser manejados y controlados durante la práctica profesional (O'Connor, 2020). Los estudiantes $y$ futuros trabajadores sociales tienen que entender y manejar sus estados emocionales, permitiéndoles tanto el afrontamiento de sus propias frustraciones como constituirse en referentes de los usuarios a la hora de imponerse a sus dificultades.

\section{Referencias bibliográficas}

APA. (2019). Ethical principles of psychologists and code of conduct. http://www.apa.org/ ethics/code/index.aspx

Augusto, J. M., Aguilar-Luzón, M. C. y Salguero, M. F. (2008). El papel de la IEP y del Optimismo/Pesimismo disposicional en la resolución de problemas sociales: Un estudio con alumnos de trabajo social. Electronic Journal of Research in Educational Psychology, 6(15), 363-382.

Augusto-Landa, J. M., Pulido-Martos, M. y LopezZafra, E. (2011). Does Perceived Emotional Intelligence and Optimism/pessimism Predict Psychological Well-being? Journal of Happiness Studies, 12(3), 463-474. https://doi. org/10.1007/s10902-010-9209-7

Bae, J., Jennings, P. F., Hardeman, C. P., Kim, E., Lee, M., Littleton, T. y Saasa, S. (2020). Compassion Satisfaction Among Social Work Practitioners: The Role of Work-Life Balance. Journal of Social Service Research, 46(3), 320-330. https://doi.org/10.1080/01488376.2 019.1566195

Bedoya-Gallego, D. M., Buitrago-Duque, D. C. y Vanegas-Arbeláez, A. A. (2019). Transdisciplinariedad en salud mental: Una propuesta de modelo de formación. Interdisciplinaria, 36(1), 119-132. https://doi.org/10.16888/ interd.36.1.9
Blakemore, T. y Agllias, K. (2019). Student Reflections on Vulnerability and Self-awareness in a Social Work Skills Course. Australian Social Work, 72(1), 21-33. https://doi.org/10.1 080/0312407X.2018.1516793

Borrego-Alés, Y., Vázquez-Aguado, O. y Orgambídez-Ramos, A. (2020). Analysis of the Occupational Health of a Group of Social Workers. En J. L. Sarasola, F. Maturo y Š. Hošková-Mayerová (Eds.), Qualitative and Quantitative Models in Socio-Economic Systems and Social Work (pp. 63-72). Switzerland: Springer International Publishing.

Bunce, L., Lonsdale, A. J., King, N., Childs, J. y Bennie, R. (2019). Emotional Intelligence and Self-Determined Behaviour Reduce Psychological Distress: Interactions with Resilience in Social Work Students in the UK. British Journal of Social Work, 49, 2092-2111. https:// doi.org/10.1093/bjsw/bcz008

Callea, A., De Rosa, D., Ferri, G., Lipari, F. y Costanzi, M. (2019). Are More Intelligent People Happier? Emotional Intelligence as Mediator between Need for Relatedness, Happiness and Flourishing. Sustainability, 11, 1022. https://doi.org/10.3390/su11041022

Caravaca, F., Carrión, J. y Pastor, E. (2018). Síndrome de burnout y satisfacción laboral en profesionales del trabajo social en prisiones de España. Revista Española de Sanidad Penitenciaria, 20, 40-47.

Cejudo, J., López-Delgado, M. L. y Rubio, M. J. (2016). Inteligencia emocional y resiliencia: su influencia en la satisfacción con la vida en estudiantes universitarios. Anuario de Psicología, 46(2), 51-57. https://doi.org/10.1016/j. anpsic.2016.07.001

Chamorro-Premuzic, T., Bennett, E. y Furnham, A. (2007). The happy personality: Mediational role of trait emotional intelligence. Personality and Individual Differences, 42, 1633-1639. https://doi.org/10.1016/j.paid.2006.10.029

Cohen, J. (1988). Statistical power analysis for the behavioral sciences $\left(2^{\mathrm{a}}\right)$. Hillsdale, NJ: Erlbaum.

Cook, L. L. (2020). The home visit in child protection social work: Emotion as resource and risk 
for professional judgement and practice. Child y Family Social Work, 25, 18-26. https://doi. org $/ 10.1111 /$ cfs. 12647

Crowder, R. y Sears, A. (2017). Building Resilience in Social Workers: An Exploratory Study on the Impacts of a Mindfulness-based Intervention. Australian Social Work, 70(1), 17-29. https:// doi.org/10.1080/0312407X.2016.1203965

Diener, E., Suh, E. M., Lucas, R. E. y Smith, H. L. (1999). Subjective Weil-Being: Three Decades of Progress. Psychological Bulletin, 125(2), 276-302.

Dore, I. (2019). Talking about emotion: how are conversations about emotion enabled in the context of social work practice education? Social Work Education, 38(7), 846-860.

Esteban, B. (2014). Una aproximación a la influencia de la Inteligencia Emocional Percibida en su relación con los niveles de Burnout y Engagement en el desempeño del Trabajo Social. Azarbe, 3, 123-131.

Esteban-Ramiro, B. y Fernández-Montaño, P. (2017). Las competencias para el desempeño profesional en Trabajo Social dentro de la formación: del burnout al engagement a través del manejo de las emociones. Trabajo Social Global, 7(13), 142-168.

Extremera, N., Durán, A. y Rey, L. (2005). La inteligencia emocional percibida y su influencia sobre la satisfacción vital, la felicidad subjetiva y el "engagement" en trabajadores de centros para personas con discapacidad intelectual. Ansiedad y Estrés, 11(1), 63-73.

Extremera, N. y Fernández-Berrocal, P. (2014). The Subjective Happiness Scale: Translation and Preliminary Psychometric Evaluation of a Spanish Version. Social Indicators Research, 119(1), 473-481. https://doi.org/10.1007/ s11205-013-0497-2

Extremera, N. y Rey, L. (2016). Ability emotional intelligence and life satisfaction: Positive and negative affect as mediators. Personality and Individual Differences, 102, 98-101. https:// doi.org/10.1016/j.paid.2016.06.051

Extremera, N., Salguero, J. M. y Fernández-Berrocal, P. (2011). Trait Meta-Mood and Subjective Happiness: A 7-week Prospective Study.
Journal of Happiness Studies, 12(3), 509-517. https://doi.org/10.1007/s10902-010-9233-7

Fernández-Berrocal, P. y Extremera, N. (2009). La Inteligencia Emocional y el estudio de la felicidad. Revista Interuniversitaria de Formación Del Profesorado, 23(3), 85-108.

Fernandez-Berrocal, P., Extremera, N. y Ramos, N. (2004). Validity and Reliability of the Spanish Modified Version of the Trait Meta-Mood Scale. Psychological Reports, 94(3), 751-755. https://doi.org/10.2466/pr0.94.3.751-755

Galarza, A. L., Castañeiras, C. E. y Fernández, M. (2018). Predicción de comportamientos suicidas y autolesiones no suicidas en adolescentes argentinos. Interdisciplinaria, 35(2), 307-326. https://doi.org/10.16888/ interd.2018.35.2.4

Golightley, M. y Kirwan, G. (2019). Social Work and Mental Health. En K. O'Donoghue y R. Munford (Eds.), New Theories for Social Work Practice: Ethical Practice for Working with Individuals, Families and Communities (pp. 100-116). London: Jessica Kingsley.

Gómez-Romero, M. J., Limonero, J. T., Toro, J., Montes-Hidalgo, J. y Tomás-Sábado, J. (2018). Relación entre inteligencia emocional, afecto negativo y riesgo suicida en jóvenes universitarios. Ansiedad y Estrés, 24(1), 18-23. https:// doi.org/10.1016/j.anyes.2017.10.007

Grant, L. y Kinman, G. (2012). Enhancing Wellbeing in Social Work Students: Building Resilience in the Next Generation. Social Work Education, 31(5), 605-621. https://doi.org/10. 1080/02615479.2011.590931

Grant, L., Kinman, G. y Alexander, K. (2014). What's All this Talk About Emotion? Developing Emotional Intelligence in Social Work Students. Social Work Education, 33(7), 874-889. https://doi.org/10.1080/02615479.2 014.891012

Grootegoed, E. y Smith, M. (2018). The Emotional Labour of Austerity: How Social Workers Reflect and Work on Their Feelings towards Reducing Support to Needy Children and Families. The British Journal of Social Work, 48(7), 1929-1947. https://doi.org/10.1093/ bjsw/bcx 151 
Guerra-Bustamante, J., León-del-Barco, B., Yuste-Tosina, R., López-Ramos, V. M. y Mendo-Lázaro, S. (2019). Emotional Intelligence and Psychological Well-Being in Adolescents. International Journal of Environmental Research and Public Health, 16(10), 1720. https://doi.org/10.3390/ijerph16101720

Ibrahim, H. B. H., Jarimal, N., Nawi, N. H. y Ahmad, P. H. M. (2018). Emotions, Emotional Intelligence and Professional Practice in Caring Professions. Asian Social Work Journal, 3(1), 35-45.

Ingram, R. (2013). Locating Emotional Intelligence at the Heart of Social Work Practice. British Journal of Social Work, 43(5), 987-1004. https://doi.org/10.1093/bjsw/ bcs029

López, E. y Chaparro, M. Y. (2006). Competencias laborales del trabajador social vistas desde el mercado laboral. Tabula Rasa, 5, 261-293. https://doi.org/10.25058/20112742.278

Lyubomirsky, S. y Lepper, H. S. (1999). A measure of subjective happiness: Preliminary reliability and construct validation. Social Indicators Research, 46(2), 137-155.

Mayer, J. D., Salovey, P. y Caruso, D. R. (2008). Emotional intelligence: New ability or eclectic traits? American Psychologist, 63(6), 503-517. https://doi.org/10.1037/0003-066X.63.6.503

Mikulic, I. M., Crespi, M. y Radusky, P. (2015). Construcción y validación del Inventario de Competencias Socioemocionales para Adultos (ICSE). Interdisciplinaria, 32(2), 307-330. https://doi.org/10.16888/ interd.2015.32.2.7

Millán-Franco, M., Gómez-Jacinto, L., Hombrados-Mendieta, I., García-Martín, M. A. y García-Cid, A. (2019). Influence of time of residence on the sense of community and satisfaction with life in immigrants in Spain: The moderating effects of sociodemographic characteristics. Journal of Community Psychology, 47(5), 1078-1094. https://doi.org/10.1002/ jcop. 22172

Montagud, X. (2016). Las consecuencias de la burocratización en las organizaciones de servicios sociales. Comunitania. Revista Interna- cional de Trabajo Social y Ciencias Sociales, 11, 69-89. https://doi.org/10.5944/comunitania.11.4

Morilla-Luchena, A., Borrego-Alés, Y., Orgambídez-Ramos, A. y Vázquez-Aguado, O. (2019). Aspectos psicosociales y calidad de vida laboral en los/as profesionales de la intervención social. Prisma Social, 26, 131-158.

Morrison, T. (2007). Emotional Intelligence, Emotion and Social Work: Context, Characteristics, Complications and Contribution. British Journal of Social Work, 37, 245-263. https:// doi.org/10.1093/bjsw/bc1016

NASW. (2017). Code of ethics of the National Association of Social Workers. https://www. socialworkers.org/About/Ethics/Code-ofEthics/Code-of-Ethics-English

O'Connor, L. (2020). How social workers understand and use their emotions in practice: A thematic synthesis literature review. Qualitative Social Work, 19(4) 645-662. https://doi. org/10.1177/1473325019843991

Prada-Ospina, R. (2019). Social psychological factors and their relation to work-related stress as generating effect of burnout. Interdisciplinaria, 36(2), 39-53. https://doi.org/10.16888/ interd.2019.36.2.3

Pulido, F. y Herrera, F. (2018). Predictores de la Felicidad y la Inteligencia Emocional en la Educación Secundaria. Revista Colombiana de Psicología, 27(1), 71-84. https://doi. org/10.15446/rcp.v27n1.62705

Ruiz-Aranda, D., Extremera, N. y Pineda-Galán, C. (2014). Emotional intelligence, life satisfaction and subjective happiness in female student health professionals: the mediating effect of perceived stress. Journal of Psychiatric and Mental Health Nursing, 21(2), 106-113. https://doi.org/10.1111/jpm.12052

Sabater, C., De Armas, D. y Cabezas, P. (2019). La satisfacción laboral de los trabajadores sociales en La Rioja de acuerdo con la teoría bifactorial de Herzberg. Cuadernos de Trabajo Social, 32(2), 397-405. https://doi.org/10.5209/ cuts. 58635

Salovey, P. y Mayer, J. D. (1990). Emotional Intelligence. Imagination, Cognition and Person- 
ality, 9(3), 185-211.

Salovey, P., Mayer, J. D., Goldman, S. L., Turvey, C. y Palfai, T. P. (1995). Emotional attention, clarity, and repair:exploring emotional intelligence using the Trait Meta-Mood Scale. En J. W. Pennebaker (Ed.), Emotion, disclosure, \& health (pp. 125-154). https://doi. org/10.1037/10182-006

Samper-García, P., Mesurado, B., Richaud, M. C. y Llorca, A. (2016). Validación del cuestionario de conciencia emocional en adolescentes españoles. Interdisciplinaria, 33(1), 163-176. https://doi.org/10.16888/interd.2016.33.1.10

Sánchez-Álvarez, N., Extremera, N. y Fernández-Berrocal, P. (2016). The relation between emotional intelligence and subjective wellbeing: A meta-analytic investigation. The Journal of Positive Psychology, 11(3), 276-285. https://doi.org/10.1080/17439760.2 015.1058968

Sewell, K. M. (2020). Examining the Place of Emotions, Affect, and Regulation in Social Work Education. Journal of Social Work Education, 56, 5-16. https://doi.org/10.1080/1 0437797.2019 .1627262

Sheppard, M. y Charles, M. (2015). Head and Heart: An Examination of the Relationship between the Intellectual and Interpersonal in Social Work. British Journal of Social Work, 45(6), 1837-1854. https://doi.org/10.1093/ bjsw/bcu117

Sheppard, M., Charles, M., Rees, P., Wheeler, M. y Williams, R. (2018). Inter-Personal and Critical-Thinking Capabilities in Those about to Enter Qualified Social Work: A Six-Centre Study. British Journal of Social Work, 48(7), 1855-1873. https://doi.org/10.1093/bjsw/ bcx143

Soto-Rosales, A. y González-Losada, S. (2018). Satisfacción laboral y desgaste profesional en trabajadores de servicios sociales de atención a la infancia. Trabajo Social Global, 8(14), 80-107. https://doi.org/10.30827/tsg-gsw. v8i14.7222

Stanley, S. y Bhuvaneswari, G. M. (2016). Reflective ability, empathy, and emotional intelligence in undergraduate social work students: a cross-sectional study from India. Social Work Education, 35(5), 560-575. https://doi.org/10. 1080/02615479.2016.1172563

Stanley, S. y Bhuvaneshwari, M. G. (2018). Personality Attributes of Social Work Students: An Assessment of Empathy, Emotional Intelligence, and Resilience. Social Work Chronicle, 7(1), 85-110.

Stanley, S., Buvaneswari, G. M. y Meenakshi, A. (2020). Predictors of empathy in women social workers. Journal of Social Work, 20(1), 43-63. https://doi.org/10.1177/1468017318794280

Urien, B., Díez, V. y Osca, A. (2019). Justicia, satisfacción y conductas contraproductivas: un estudio con trabajadoras y trabajadores sociales basado en la teoría de las demandas y de los recursos laborales (JD-R). Cuadernos de Trabajo Social, 32(1), 141-152. https://doi. org/10.5209/CUTS.56882

Vallellano, M. D. y Rubio-Valdehita, S. (2018). Carga mental y satisfacción laboral: un estudio comparativo entre trabajadoras sociales, educadoras sociales y profesoras de enseñanza primaria. Ansiedad y Estrés, 24(2-3), 119-124. https://doi.org/10.1016/j.anyes.2018.08.003

Ye, J., Yeung, D. Y., Liu, E. S. C. y Rochelle, T. L. (2019). Sequential mediating effects of provided and received social support on trait emotional intelligence and subjective happiness: A longitudinal examination in Hong Kong Chinese university students. International Journal of Psychology, 54(4), 478-486. https://doi.org/10.1002/ijop.12484

Recibido: 10 de diciembre de 2019 Aceptado: 29 de marzo de 2021 